\title{
Spinal Cord Injuries
}

\author{
Surg Lt Cdr M Malhotra*, Brig HS Bhatoe, vsM $^{+}$, Col SM Sudambrekar"
}

\begin{abstract}
Spinal Cord injury (SCI) is one of the most devastating and demoralizing ailment for both the patient and the medical practitioner. However, with the better understanding of the pathophysiology, better imaging modalities and emphasis on immobilization and rehabilitation has provided a ray of hope to such patients. The initial care aims at immobilization and evacuation by the classical log roll method and focuses on life-saving procedures. Basic imaging should be augmented with an MRI in doubtful cases. Immobilization either external or internal should be followed by early efforts for rehabilitation. The use of steroids during the acute phase has become controversial. The focus of latest studies has shifted to neuroprotective and regenerative agents.
\end{abstract}

MJAFI 2010; 66 : 325-328

Key Words : Spinal cord injury; Neurological assessment; Magnetic resonance imaging; Methylprednisolone

\section{Introduction}

$\mathrm{D}$ uring ancient times, spinal trauma and paralysis was untreatable or fatal and considered 'An ailment not to be treated' [1]. Even in 1805, Admiral Nelson recognized the severity of his spinal injury and his imminent death after he was paralyzed during the Battle of Trafalgar. The other famous personalities who suffered from spinal cord injury include President James A. Garfield, General George Patton and Christopher 'Superman' Reeve. Thankfully, major advances over the past 50 to 60 years in the medical, surgical, and rehabilitation fields now allow spinal cord injury (SCI) patients to lead long and productive lives.

However, SCI remains a significant cause of disability worldwide despite vast improvements in conventional therapies. This tragedy is compounded by the fact that combat SCI results not only in loss of trained manpower but also renders these young combatants permanently disabled. This article attempts to provide a glimpse into current therapies for SCI and the future of these new therapies and diagnostic imaging methodologies.

\section{Pathophysiology}

Cases of SCI that involve gunshot or stab injuries (sharp trauma) may result in complete severing of the spinal cord, but in most cases patients present with an anatomically incomplete lesion of the spinal cord with compression or contusion injury being more frequent.

SCI has been described as developing in three phases: acute, secondary, and chronic. The direct mechanical trauma to the spinal cord is quickly followed by local ischemia and hemorrhage, resulting in edema and neural cell death in the immediate vicinity [2]. The term spinal shock was introduced to describe a patient's condition following acute SCI: muscle paralysis, flaccid muscle tone, and loss of tendon reflexes below the level of the lesion [3].

Secondary injury is characterized by dramatic ionic changes in the injury zone after SCI which results in the breakdown of cellular membranes and the release of inflammatory mediators [4].

In the chronic phase of SCI a cystic cavity forms that is surrounded by an astrocytic scar. In addition, there is degeneration of an axon distal to an injury, as was first described by Waller (Wallerian degeneration) [5]. Long term sequelae following SCI, include chronic pain and spasticity.

\section{Epidemiology}

Males are disproportionately affected with a 4:1 maleto-female ratio, and the majority of injuries occur between the ages of 16 and 30 [6].

Spine injuries usually occur in the most mobile spinal segments: the cervical spine, and the thoracolumbar spine. In the cervical spine $25 \%$ of all injuries occur in the upper cervical spine (Oc-C2), while $75 \%$ occur in the subaxial region (C3-C7). Multiple level spine injuries have been estimated to occur in 4 to $20 \%$ of all cases. In the thoracolumbar spine the anatomic distribution of injury is most common around the thoracolumbar junction

${ }^{*}$ Clinical Tutor, Department of Surgery, Armed Forces Medical College, Pune-40. ${ }^{+}$Consultant, "Senior Advisor (Surgery \& Neurosurgery), Command Hospital (Southern Command), Pune-40.

E-mail : drmunishmalhotra@gmail.com 
with L1 accounting for $16 \%$ of all injuries. Approximately, 5 to $20 \%$ of patients with thoracolumbar spine injuries have noncontiguous fractures.

\section{Initial care}

The primary efforts in the initial phase are focused on life-saving procedures. The ATLS ${ }^{\circledast}$ protocol has the broadest distribution to capture all injuries in the mostly unconscious patients [7].

Since hypotension and ischemia-reperfusion are known factors for secondary injury, step B and C of the ATLS $^{\circledR}$ protocol i.e early oxygenation and aggressive volume replacement is highly important [8].

The ATLS ${ }^{\circledR}$-protocol also emphasizes the "log roll" in step "E" [9]. This is the most critical step in the management of combat SCI as there is an immediate requirement to transfer the patient to higher level of care. Instability of the spinal cord must be presumed in any patient with complaints of a sense of instability, vertebral column pain, tenderness in the midline over the spinous processes, any neurologic deficit and suspected but not proven injury.

In cases of penetrating injuries to the spine eg gunshot wounds, the local management should only be done after adequate resuscitation and the ABCDE of the ATLS protocol. In case of CSF leak, the wound should be sutured after adequate debridement and irrigation and the causalty started on broad spectrum antibiotics. In neurologically stable patients with fragments in the cervical canal, evacuation with adequate immobilization and delaying surgery for 7-10 days makes dural repair considerably easier and reduces problems with leak.

\section{Neurological Examination}

Classification of SCI is based upon a standardized examination conducted according to the methods described in the International Standards for Neurological Classification of SCI [10] and the accompanying Reference Manual [11]. The neurological assessment for traumatic SCI involves three parts: the sensory, motor, and rectal ("sacral sparing") examinations.

With information obtained from the examination described above, one is able to classify the level and

Associated trauma with spinal cord injury [7]

$\begin{array}{lc}\text { Injury type } & \text { Occurrence (\%) } \\ \text { No additional injury } & 55.8 \\ \text { Fractures of the trunk } & 17.2 \\ \text { Long bone fractures } & 13.9 \\ \text { Head and face trauma } & 13.8 \\ \text { Pneumothorax and chest injury } & 8.8 \\ \text { Abdominal injury } & 8.6\end{array}$

severity of injury. The neurological level of injury (NLI) is defined as the most caudal segment of the spinal cord with normal sensory and motor function on both sides of the body. The zone of partial preservation (ZPP) refers to those dermatomes and myotomes caudal to the NLI that remain partially innervated and demonstrate partial preservation of function. Severity of injury is graded by the ASIA Impairment Scale (AIS), a 5-point ordinal scale from A to $\mathrm{E}$ (Tables 1,2).

A complete injury is defined by the absence of sacral sparing namely no sensation in the S4-5 segment or the inability to voluntarily contract the anal sphincter.

\section{Imaging}

The primary diagnostics include AP and Lateral radiograph of the spine [12]. Additional anterior cervical spine and odontoid axis films may be required $[13,14]$.

At a higher level of care, severity of SCI is accurately assessed by magnetic resonance imaging (MRI). Characteristics shown to be related to prognosis have included the presence of intramedullary hemorrhage, presence of edema, length of edema, and cord compression [15]. In addition to the diagnosis of SCI, findings on MRI are related to neurological recovery and functional capabilities following SCI [15,16].

\section{Management}

The management in the acute setting can be divided into

1. Efforts to treat and impact outcomes following acute SCI

2. Management of acute medical conditions.

\section{Efforts to Treat and Impact Outcomes following Acute SCI}

Only methylprednisolone is routinely administered in the acute clinical setting. Presently, efforts have focused on (a) rehabilitative strategies (b) optimizing medical management to prevent secondary complications.

\section{Table 2}

The ASIA impairment scale [11]

\section{Grade Definition}

A Complete. No sensory or motor function is preserved in the sacral segments S4-S5.

B Incomplete. Sensory but not motor function is preserved below the neurological level and includes the sacral segments S4-5.

C Incomplete. Motor function is preserved below the neurological level, and more than half of the key muscles below the neurological level have a muscle grade $<3$ (grades 0 to 2 ).

D Incomplete. Motor function is preserved below the neurological level, and at least half of the key muscles below the neurological level have a muscle grade $\geq 3$.

E Normal. Sensory and motor functions are normal. 


\section{Steroids}

For the treatment of traumatic SCI, high-dose methylprednisolone (MP) therapy is the only pharmacological therapy reported to have efficacy in phase III randomized trials. [17]. It is indicated if the patient presents with a neurological deficit within 8 hours of injury and is administered as an intravenous bolus (30 mg/kg) over 15 minutes followed by a 45 -minute pause then a continuous drip $(5.4 \mathrm{mg} / \mathrm{kg} / \mathrm{h})$ for 23 hours $[17,18]$.

\section{Management of Acute Medical Conditions}

\section{a) Deep Vein Thromboses (DVT) and Pulmonary Emboli (PE)}

The occurrence of DVT and PE are among the most feared complications during the acute period. Without prophylaxis, $47-100 \%$ of individuals with acute SCI will develop a DVT (19), and PE is the third leading cause of death [19]. Pharmacologic prophylaxis should be initiated as soon as possible, preferably within 72 hours of injury.

\section{b) Autonomic Dysreflexia (AD)}

Patients with a neurological level at and above T6 are also at risk for a life-threatening complication called autonomic dysreflexia. It is characterized by the abrupt onset of malignant hypertension following a noxious stimulus.

\section{c) Respiratory Complications}

Overall, respiratory complications are the leading cause of mortality during the first year post injury as well as during long-term follow-up.

\section{d) Pressure ulcers}

These are a devastating complication that occurs in up to $40 \%$ of SCI patients during the initial hospitalization.

\section{e) Bowel Dysfunction}

Because of autonomic dysfunction and immobility, gut transit times are prolonged, which in turn can predispose to severe constipation or impaction. In addition, volitional control of defecation is often absent with severe injuries.

\section{f) Bladder dysfunction}

Urinary retention is the primary voiding abnormality during the acute phase of SCI. It is prudent to maintain an indwelling catheter until the patient is transferred to a rehabilitation setting.

\section{Surgical Treatment of Spinal Injury}

Surgical treatment can range from simple limitation in activity to external orthosis to open reduction and internal fixation with spinal instrumentation. The goal of treating these injuries is to utilize the least invasive surgical technique to stabilize the injured segment while limiting the potential for subsequent catastrophic neurologic injury, progression of a deformity, and chronic pain conditions.

\section{a) External Orthosis}

Numerous external orthosis (spinal braces) options are available for the treatment of spinal injuries. For the cervical spine, options ranging from least to most restrictive are soft and hard cervical collars (Philadelphia, Aspen, Miami J), cervical bracing with the addition of a thoracic vest (SOMI and Minerva braces), and halovest immobilization.

In the thoracic spine, the rib cage provides some natural support for thoracic spine fractures. The upper thoracic region (T5 and above) is a very difficult region to immobilize with external orthosis, unless the patient is immobilized with a halo orthosis with a long thoracic vest.

Spinal fractures from T6 to L2 are typically braced with a custom molded, hard-shell orthosis (thoracolumbar-sacral orthosis (TLSO)) or with more versatile, adjustable-fit braces (e.g., Jewitt, Aspen) or clamshell brace.

Below L3, a lumbosacral orthosis is used for support. In addition, to increase the immobilization at the lumbosacral junction, a leg extension can be fitted to the orthosis to assist in limiting motion across the pelvis [20].

\section{b) Surgical Options for Traumatic SCI}

Controversy persists in the surgical community regarding the optimal treatment of many traumatic spinal injuries, especially regarding timing of surgical intervention and type of surgical approach. Surgical intervention is often advocated to (a) decompress the neural elements; (b) prevent possible late neurologic injury; (c) correct and prevent deformity; and (d) provide for early mobilization [20].

Spinal instrumentation is a method of straightening and stabilizing the spine after spinal fusion, by surgically attaching hooks, rods, and wire to the spine in a way that redistributes the stresses on the bones. Anterior (ventral), posterior (dorsal), and combined anterior and posterior approaches can be used to treat traumatic spinal instability. Anterior approaches may be favored in situations where a herniated disc or bone fragment is causing ventral compression on the spinal cord or in fracture patterns where the integrity of the anterior column of the spine is significantly compromised [20].

Posterior surgical approaches and instrumentation typically allow for better reduction when deformities are present and may benefit in restoring the posterior tension 
band in distraction-type injuries where there is disruption of the posterior ligamentous structures [20].

In translation injuries (fracture-dislocations), when there is severe, circumferential disruption of the spinal column, combined anterior-posterior instrumentation procedures may be used.

Intraoperative imaging is often used by surgeons to confirm the appropriate level for surgery and improve the accuracy of spinal instrumentation placement.

\section{Future Trends}

Research scientists at the animal laboratory level have developed multiple strategies for studying and repairing the injured spinal cord including both acute and chronic subjects. These strategies include [21]:

a) Reduce the effects of the damage

b) Encourage correct neuron function/connections via a nerve bridge

c) Enhance regeneration/axon growth

d) Replace lost nerve cells

e) Inhibit scar/gliosis formation

f) Reduce neurocircuit deficits

\section{Conclusion}

The care of individuals following traumatic SCI has improved dramatically since pre-World War II when mortality was $60-80 \%$ within months of injury. This has been facilitated largely by the development of specialized SCI centers predicated on providing interdisciplinary, comprehensive care. With proper medical management, secondary complications are largely preventable and many individuals now lead healthy, productive lives following injury. Unfortunately, the extent of neurorecovery following the most severe injuries remains limited, and rehabilitation to a large extent focuses on teaching compensatory strategies to deal with resulting impairments. Although the number of clinical trials has been limited to date, the future holds great promise.

\section{Conflict of Interest}

None identified

\section{References}

1. Goodrich JT. History of spine surgery in the ancient and medieval worlds. Neurosurg Focus 2004; 16: E2.

2. Kwon BK, Tetzlaff W, Grauer JN. Pathophysiology and pharmacologic treatment of acute spinal cord injury. Spine J 2004; 4: 451-64.

3. Hiersemenzel LP, Curt A, Dietz V. From spinal shock to spasticity: neuronal adaptations to a spinal cord injury. Neurology 2000; 54: 1574-82.

4. Schadt JC, Barnes CD. Motoneuron membrane changes associated with spinal shock and the Schiff-Sherrington phenomenon. Brain Res 1980; 201: 373-83.

5. Waller A. Experiments on the section of glossopharyngeal and hypoglossal nerves of the frog and observations of the alternatives produced thereby in the structure of their primitive fibres. Phil Trans R Soc Lond 1850; 140: 423-9.

6. National Spinal Cord Injury Statistical Center. Spinal Cord Injury: Facts and Figures at a Glance. 2005; 36.

7. Committee ACoS. Advanced Trauma Life Support (ATLS) for Doctors, Chicago/IL. 7. 2004.

8. Baptiste DC, Fehlings MG. Update on the treatment of spinal cord injury. Prog Brain Res 2007; 161: 217-33.

9. Holly LT, Kelly DF, Counelis GJ, Blinman T, McArthur DL, Cryer HG. Cervical spine trauma associated with moderate and severe head injury: incidence, risk factors, and injury characteristics. J Neurosurg 2002; 96: 285-91.

10. American Spinal Injury Association. International Standards for the Neurological Classification of Spinal Cord Injury. Chicago: American Spinal Injury Association 2002.

11. American Spinal Injury Association. Reference Manual for the International Standards for Neurological Classification of Spinal Cord Injury. Chicago: American Spinal Injury Association 2003.

12. Linsenmaier U, Kanz KG, Mutschler W, Pfeifer KJ. Radiological diagnosis in polytrauma: interdisciplinary management. Rofo 2001; 173: 485-93.

13. Morris CG, McCoy E. Clearing the cervical spine in unconscious polytrauma victims, balancing risks and effective screening. Anaesthesia 2004; 59: 464-82.

14. Platzer P, Hauswirth N, Jaindl M, Chatwani S, Vecsei V, Gaebler C. Delayed or missed diagnosis of cervical spine injuries. J Trauma 2006; 61: 150-5.

15. Flanders AE, Spettell CM, Friedman DP. The relationship between the functional abilities of patients with cervical spinal cord injury and the severity of damage revealed by MR imaging. Am J Neuroradiol 1999; 20: 926-34.

16. Stroman PW, Kornelsen J, Bergman A. Noninvasive assessment of the injured human spinal cord by means of functional magnetic resonance imaging. Spinal Cord 2004; 42: 59-66.

17. Bracken MB, Shepard MJ, Collins WF. A randomized, controlled trial of methylprednisolone or naloxone in the treatment of acute spinal-cord injury: results of the Second National Acute Spinal Cord Injury Study. N Engl J Med 1990; 322: 1405-11.

18. Coleman WP, Benzel D, Cahill DW. A critical appraisal of the reporting of the National Acute Spinal Cord Injury Studies (II and III) of methylprednisolone in acute spinal cord injury. J Spinal Disord 2000; 13: 185-99.

19. Geerts WH, Pineo GF, Heit JA. Prevention of venous thromboembolism: the seventh ACCP conference on antithrombotic and thrombolytic therapy. Chest 2004; 126 (Suppl): 338S-400S.

20. Schwartz, Eric D, Flanders, Adam E. Title: Spinal Trauma: Imaging, Diagnosis, and Management, 1st Edition, 2007 Lippincott Williams and Wilkins.

21. Spinal Cord Injury - Past, Present, and Future: William H Donovan, MD; J Spinal Cord Med 2007; 30: 85-100. 